\title{
Article \\ UDP-Glucosyltransferases Induced by Nosema bombycis Provide Resistance to Microsporidia in Silkworm (Bombyx mori)
}

\author{
Bin $Y u^{1}{ }^{1}$, Qiuhua Yang ${ }^{1}$, Junhong Wei ${ }^{1,2}$, Guoqing Pan ${ }^{1,2}$, Chunfeng $\mathrm{Li}^{1,2, *}$ and Zeyang Zhou ${ }^{1,2,3, *(1)}$ \\ 1 State Key Laboratory of Silkworm Genome Biology, Southwest University, Chongqing 400715, China; \\ yubin5868@outlook.com (B.Y.); YQH17320362547@163.com (Q.Y.); weijunhong@swu.edu.cn (J.W.); \\ gqpan@swu.edu.cn (G.P.) \\ 2 Chongqing Key Laboratory of Microsporidia Infection and Control, Southwest University, \\ Chongqing 400715, China \\ 3 College of Life Sciences, Chongqing Normal University, Chongqing 401331, China \\ * Correspondence: licf@swu.edu.cn (C.L.); zyzhou@swu.edu.cn (Z.Z.)
}

check for updates

Citation: Yu, B.; Yang, Q.; Wei, J.; Pan, G.; Li, C.; Zhou, Z. UDP-Glucosyltransferases Induced by Nosema bombycis Provide Resistance to Microsporidia in Silkworm (Bombyx mori). Insects 2021, 12, 799. https://doi.org/10.3390/ insects12090799

Academic Editor: Maurizio Francesco Brivio

Received: 12 August 2021 Accepted: 4 September 2021 Published: 7 September 2021

Publisher's Note: MDPI stays neutra with regard to jurisdictional claims in published maps and institutional affiliations.

Copyright: (C) 2021 by the authors. Licensee MDPI, Basel, Switzerland. This article is an open access article distributed under the terms and conditions of the Creative Commons Attribution (CC BY) license (https:// creativecommons.org/licenses/by/ $4.0 /)$.
Simple Summary: Nosema bombycis (N. bombycis), an obligate intracellular eukaryotic parasite, is a virulent pathogen of the silkworm, that causes major economic losses. Although many studies have reported on $B$. mori host response to this pathogen, little is known about which genes are induced by $N$. bombycis. Our results showed that two B. mori uridine diphosphate-glucosyltransferases (UGTs) (BmUGT10295 and BmUGT8453) could be activated by N. bombycis and provide resistance to the microsporidia in silkworms. These results will contribute to our understanding of host stress reaction to pathogens and the two pathogen-induced resistant genes will provide a target for promoting pathogen resistance.

Abstract: As a silkworm pathogen, the microsporidian N. bombycis can be transovarially transmitted from parent to offspring and seriously impedes sericulture industry development. Previous studies found that Uridine diphosphate (UDP)-glycosyltransferases (UGTs) are involved in regulating diverse cellular processes, such as detoxification, pigmentation, and odorant sensing. Our results showed that BmUGT10295 and BmUGT8453 genes were specifically induced in infected silkworms, but other BmUGTs were not. Tissue distribution analysis of the two BmUGTs showed that the transcriptions of the two BmUGTs were mainly activated in the midgut and Malpighian tubule of infected silkworms. Furthermore, there were significantly fewer microsporidia in over-expressed BmUGTs compared with the control, but there were significantly more microsporidia in RNA interference BmUGTs compared with the control. These findings indicate that the two BmUGTs were induced by N. bombycis and provided resistance to the microsporidia.

Keywords: Bombyx mori; innate immunity; UDP-glucosyltransferase; induced expression

\section{Introduction}

Microsporidia are a group of obligate intracellular parasites that can infect nearly all animal phyla in nature [1,2]. Microsporidia infection can cause major economic losses; for example, Nosema ceranae causes serious disease in adult honey bees [3,4], Enterocytozoon hepatopenaei affects cultured shrimp [5,6], and Cryptosporidium baileyi impacts the digestive and / or respiratory tract of many bird species across various orders $[7,8]$. Moreover, infection with some microsporidia is a serious threat to human life and health [9-11]. Although there are many studies on microsporidia, there has not been sufficient research on host response because of the complexity of host-pathogen interactions and the differences among species [12-14]. The microsporidian N. bombycis was identified from the silkworm, Bombyx mori, in which it causes pebrine and leads to enormous economic losses in the silkworm industry [15]. Upon infection, innate immune responses of silkworms 
against the pathogen N. bombycis are activated, such as hemocytin [16] and Bombyx Turtle protein expression [17]. Moreover, the autophagy pathway is induced by N. bombycis infection $[18,19]$, and apoptosis and reactive oxygen species production also change upon infection with $N$. bombycis [20].

Uridine diphosphate (UDP)-glycosyltransferases (UGTs) are found in all living organisms, including animals, plants, bacteria, and viruses. As a phase II enzyme in the detoxification system, UGTs catalyze the addition of sugars to a broad range of lipophilic molecules; this turns the lipophilic molecules into more water-soluble metabolites that can be easily excreted to regulate internal molecules and protect the cellular system from damage by toxic foreign compounds [21]. In insects, UGTs play a vital role in the biotransformation of exogenous and endogenous compounds from being hydrophobic to hydrophilic, which results in more efficient internal molecule regulation and excretion that prevents retention of toxic foreign compounds. For example, UGTs are involved in the detoxification of xenobiotics produced by the plants upon which they feed [22,23].

The UGTs that participate in detoxification of plant toxins can also result in crossresistance to various pesticides [24-28], and therefore could be a crucial knock down target when developing novel pest control strategies to improve the natural toxicity of plants or chemicals to pests [29]. Besides being detoxification enzymes, UGTs are also involved in physiological processes [30-32], pigmentation [33], and odorant sensing [34,35]. In B. mori, many UGTs have been identified [36,37]; BmUGT10286 catalyzes quercetin 5-O-glucoside formation, which protects pre-pupae from the harmful effects of UV radiation during metamorphosis and facilitates green cocoon formation [38]. The green cocoon of silkworms that results from quercetin 5-O-glucosyltransferase is an evolved response to dietary toxins [39]. Moreover, BmUGT1 shows activity with flavonoids, coumarins, terpenoids, and simple phenols, which supports a role of this enzyme in detoxication processes [40], and BmUGT013829, which is highly expressed in larval and adult antennae, may be involved in insect olfaction [36]. Though UGTs are involved in detoxification, pigmentation, odorant sensing and cocoon formation, there are no any reports about the involvement of UGT in microbial stress response in insects.

B. mori is an economically important insect and a lepidopteran model for investigating gene functions. In previous studies, many genes of silkworm were found to be induced by $N$. bombycis that were involved in many signal transduction pathways [19,41-44]. However, there was no report that UGT was induced in silkworm, and there was also few reports that UGT was involved in the process of biological stress. In this study, we determined that BmUGT10295 and BmUGT8453 genes were induced by N. bombycis and provided resistance to microsporidia proliferation. Our study is the first report to find that UGT facilitate resistance to pathogens in insects.

\section{Materials and Methods}

\subsection{Insect Rearing and Cell Lines}

The B. mori strain Dazao was reared on an artificial diet (Nihonnosanko, Yokohama, Japan) and maintained at $25^{\circ} \mathrm{C}$ under a photoperiod of $12 \mathrm{~h} \mathrm{light}$ and $12 \mathrm{~h}$ dark.

BmN-SWU1, a B. mori cell line, was cultured in TC-100 medium (United States Biological Inc., Swampscott, MA, USA) with $10 \%$ fetal bovine serum and $1 \%$ penicillin/streptomycin (Gibco, Waltham, MA, USA) and maintained at $28^{\circ} \mathrm{C}$ [45].

\subsection{Immune Challenge}

N. bombycis CQ1 (No. CVCC102059) was conserved in the China Veterinary Culture Collection Center. The mature spores of $\mathrm{N}$. bombycis were purified from infected silkworms and stored in sterilized distilled water at $4{ }^{\circ} \mathrm{C}$ for later use.

The artificial silkworm diet was cut into small pieces, adding $10^{6} \mathrm{~N}$. bombycis spores to each piece. Each silkworm in day 1 fifth instar was fed a small piece of artificial diet with $N$. bombycis. The silkworms that did not eat the diet within 20 min were eliminated. Control silkworms were orally administered with sterilized water. Finally, 200 silkworms 
were separately screened out and separately reared together in the infected and control groups. Three groups (six silkworms per group) were randomly selected at 3, 6, 12, 24, 48, and $72 \mathrm{~h}$ after oral challenge, and all tissues were harvested. The collected samples were stored at $-80^{\circ} \mathrm{C}$ and later used for total RNA extraction or protein extraction.

\subsection{RNA Isolation, cDNA Synthesis, and Reverse Transcription Polymerase Chain Reaction (RT-PCR)}

Total RNA isolation and cDNA synthesis were conducted as described in a previous study [46]. To investigate the transcription of BmUGT genes in B. mori, RT-PCR was performed using ExTaq (Takara, Tokyo, Japan). The PCR program using touchdown-PCR amplification was conducted under the following conditions: initial denaturation at $94{ }^{\circ} \mathrm{C}$ for $5 \mathrm{~min}$; followed by 20 cycles of $94{ }^{\circ} \mathrm{C}$ for $40 \mathrm{~s}$, annealing temperatures starting at $62{ }^{\circ} \mathrm{C}$ for $40 \mathrm{~s}$ (decreasing $0.5^{\circ} \mathrm{C} /$ cycle), and $72^{\circ} \mathrm{C}$ for $1.5 \mathrm{~min} ; 30$ cycles of $94^{\circ} \mathrm{C}$ for $1 \mathrm{~min}, 55^{\circ} \mathrm{C}$ for $40 \mathrm{~s}, 72{ }^{\circ} \mathrm{C}$ for $2 \mathrm{~min}$; and a final extension at $72{ }^{\circ} \mathrm{C}$ for $10 \mathrm{~min}$.

For RT-PCR, all BmUGT gene sequences were obtained from the InsectBase database (http:/ / www.insect-genome.com/ (accessed on 20 March 2017)) and the KAIKObase database (http:/ / sgp.dna.affrc.go.jp/KAIKObase/ (accessed on 20 March 2017)). All gene IDs are listed in Table S1. The silkworm actin3 (BmA3) gene was used as an internal control and all primer sequences are listed in Table S2. The PCR product was analyzed by $1 \%$ agarose gel electrophoresis.

\subsection{Gene Cloning}

To obtain the full-length cDNA of BmUGT10295, we used a GeneRacer ${ }^{\mathrm{TM}}$ Kit (L1502, Invitrogen, Waltham, MA, USA) to synthesize the cDNA following the kit's instructions. The primers used are listed in Table S2. PCRs were performed under the following conditions: $94{ }^{\circ} \mathrm{C}$ for $3 \mathrm{~min}$; 30 cycles of $94{ }^{\circ} \mathrm{C}$ for $30 \mathrm{~s}, 55^{\circ} \mathrm{C}$ for $30 \mathrm{~s}$, and $72{ }^{\circ} \mathrm{C}$ for $1 \mathrm{~min}$; and a final extension at $72{ }^{\circ} \mathrm{C}$ for $10 \mathrm{~min}$. The purified PCR products were inserted into the PESI-Blunt simple vector (10910, Yeasen, Shanghai, China) and the positive clones were sequenced by the Sangon Company (Shanghai, China).

To obtain the $5^{\prime}$-end sequences of BmUGT10295 according to predicted transcription initiation sites, we designed the forward primers (BmUGT10295-TSS-F; Table S2). The 5'-end sequences of BmUGT10295 were obtained by PCR using the primers BmUGT10295-TSS-F and Bm10295-race-R (Table S2). The positive bands of PCR were cut and inserted into the PESI-Blunt simple vector. Then the positive clones were sequenced by the Sangon Company.

Because the sequence of the BmUGT8453 gene in KAIKObase was full-length cDNA, the BmUGT8453 gene was also cloned and sequenced for verification. The primers used are listed in Table S2.

\subsection{Real-Time Quantitative PCR (qPCR) Analysis}

SW22934, a microarray probe of Bombyx mori eukaryotic transcription initiation factor $4 \mathrm{~A}$, was used as an internal control for normalization. The $20-\mu \mathrm{L}$ mixture included $2 \mu \mathrm{L}$ cDNA or DNA, $0.5 \mu \mathrm{L}$ of each primer (10 mM; Table S2), $10 \mu \mathrm{L}$ SYBR Green Master Mix reagent (Yeasen, Shanghai, China), and $7 \mu \mathrm{L}$ ddH ${ }_{2} \mathrm{O}$. qPCR was performed according to the following parameters: one cycle of an initial denaturation step at $95{ }^{\circ} \mathrm{C}$ for $5 \mathrm{~min}$, followed by 40 cycles at $95^{\circ} \mathrm{C}$ for $10 \mathrm{~s}, 60^{\circ} \mathrm{C}$ for $20 \mathrm{~s}$, and $72{ }^{\circ} \mathrm{C}$ for $40 \mathrm{~s}$. The relative gene expression levels were estimated according to the $2^{-\Delta \Delta \mathrm{Ct}}$ or $2^{-\Delta \mathrm{Ct}}$ method [45]. These experiments were repeated three times and all samples were run in triplicate of each time.

\subsection{Vector Constructs}

Because there were substantial sequence similarities between BmUGT10295 and BmUGT8453 (Figure S2, a partial BmUGT8453 sequence and the full-length BmUGT10295 sequence were cloned into pET32a vector to generate pET32-BmUGT10295 and pET32BmUGT8453 vectors for polyclonal antibody production. The primers are listed in Table S2.

For overexpression of the BmUGTs in BmN-SWU1 cell line, the pEHI vector was constructed. The region of the B. mori nuclear polyhedrosis virus (BmNPV) genome that 
contained homologous region 3 (HR3), which acts as an enhancer for the promoter of a nonviral gene, were cloned from BmNPV. The OpIE2-MCS-PA fragment (OpIE2 promoter, multiple cloning sites, and polyadenylation sequence) was cloned from the pIZ/V5-His vector (Invitrogen, Waltham, MA, USA). The HR3-OpIE2-MCS-PA fragment was assembled with the fragment HR3 and OpIE2-MCS-PA using overlapping PCR techniques. Then, the HR3-OpIE2-MCS-PA fragment was inserted into the PESI-Blunt simple vector (10910, Yeasen, Shanghai, China) to obtain the pEHI vector. The full-length BmUGT10295 and BmUGT8453 were cloned from the above PESI vectors. DsRed was cloned from the pDsRed2-N1 vector. Then full-length BmUGT10295, BmUGT8453 and DsRed sequences were cloned into the pEHI vector to generate pEHI-BmUGT10295, pEHI-BmUGT8453 and pEHI-DsRed vectors. The primers are listed in Table S2.

\subsection{Protein Expression, Purification, and Polyclonal Antibody Production}

The above pET32a recombinant plasmids (pET32-BmUGT10295 and pET32-BmUGT8453 vector) were transformed into BL21(DE3) cells for BmUGT expression following standard protein expression protocols. Briefly, when the culture reached an $\mathrm{OD}_{600}$ of $0.4-0.6$, it was induced with $0.1 \mathrm{mM}$ isopropyl-b-D-1-thiogalactopyranoside for $20 \mathrm{~h}$. The cells that contained recombinant vector were re-suspended in lysis buffer $(20 \mathrm{mM}$ Tris- $\mathrm{HCl}, \mathrm{pH}$ 8.0, and $100 \mathrm{mM} \mathrm{NaCl}$ ) and sonicated. Then, the fused expression proteins were purified using the Ni-NTA beads (QIAGEN, Valencia, CA, USA).

For polyclonal antibody production, all animal experiments were conducted in accordance with Laboratory Animals Ethics Review Committee of Southwest University guidelines (Chongqing, China), and the committee approved this study (Permit Number: AERCSWU2017-7). Three mice were each subcutaneously inoculated with each recombinant BmUGT10295 and BmUGT8453 (80-120 $\mu \mathrm{g}$ /mouse) homogenized with Freund's adjuvant (1:1; Sigma, St. Louis, MO, USA) four times. One week after the fourth injection, antisera were collected and stored at $-80^{\circ} \mathrm{C}$.

\subsection{Western Blotting}

The silkworm samples were ground with liquid nitrogen and then lysed with RIPA (P0013B, Beyotime Biotechnology, Shanghai, China) at $4{ }^{\circ} \mathrm{C}$ for $30 \mathrm{~min}$. The supernatants were separated by SDS-PAGE and transferred to a PVDF membrane (Roche, Basel, Switzerland). After blocking for $1 \mathrm{~h}$ at $37^{\circ} \mathrm{C}$ in TBST ( $20 \mathrm{mM}$ Tris-HCl, $150 \mathrm{mM} \mathrm{NaCl}, 0.05 \%$ Tween-20) with $5 \%(w / v)$ skim milk, membranes were incubated with 1:1000 dilutions of anti-BmUGT or negative control serum in TBST for $1 \mathrm{~h}$ at $37^{\circ} \mathrm{C}$. Following several washes, membranes were reacted with HRP-labeled goat anti-mouse IgG (Bio-Rad, Richmond, CA, USA), successively, with washing in between. ECL Plus Western Blotting Detection Reagents (Bio-Rad, Richmond, CA, USA) were used to detect the bound antibodies.

\subsection{Indirect Immunofluorescence Assay}

To detect BmUGT expression, we plated the B. mori cell line BmN-SWU1 in 6-well culture plates $\left(10^{5}\right.$ cells / well) and challenged with $N$. bombycis (spore: cell, 10:1). Then, $72 \mathrm{~h}$ after infection, infected cells were fixed in $4 \%$ paraformaldehyde and permeabilized with $0.5 \%$ Triton X-100 for $5 \mathrm{~min}$. The cells were subsequently blocked in PBS that contained $10 \%(w / v)$ goat serum and $0.5 \%(v / v)$ BSA for $1 \mathrm{~h}$ and incubated with anti-BmUGT (1:1000) for $1 \mathrm{~h}$. Alexa488 was used to label the primary antibodies and DNA was stained with DAPI (Sigma, St. Louis, MO, USA) for $30 \mathrm{~min}$. Fluorescence was observed and imaged with confocal microscopy (Olympus, Tokyo, Japan).

\subsection{0. dsRNA Synthesis}

The interference segments of BmUGT10295, BmUGT8453, and EGFP (control) were designed by the siDirect database (http:/ / sidirect2.rnai.jp/ (accessed on 30 May 2018)). T7 promoter sequences were tailed to sense and antisense primers (primers sequences in Table S2). dsRNA synthesis was conducted with a Transcript Aid T7 High Yield Transcription 
Kit (KO441, Thermo Scientific, Waltham, MA, USA), which were purified using a MicroElute RNA Clean-up Kit (R6247, OMEGA, Doraville, GA, USA) for RNA interference (RNAi).

\subsection{Overexpression and RNAi BmUGTs}

For overexpression of BmUGT10295 and BmUGT8453, BmN-SWU1 cells were transiently transfected with pEHI-BmUGT10295 or pEHI-BmUGT8453 expression plasmids $(3 \mu \mathrm{g})$, and pEHI-DsRed $(3 \mu \mathrm{g})$ was used as a control. Then, these cells were challenged with $N$. bombycis (spore: cell, 10:1).

For RNAi BmUGT10295 and BmUGT8453, BmN-SWU1 cells were transiently transfected with dsRNA of BmUGT10295 or BmUGT8453 (3 $\mu \mathrm{g})$, and the dsRNA of EGFP was used as a control. Then, these cells were challenged with $N$. bombycis (spore: cell, 10:1).

Each of the above cells was harvested on 1, 3 and 5 days post-infection N. bombycis; They were divided into two parts and broken with glass beads. One part was used for RNA extraction (R6934, OMEGA, Doraville, GA, USA) and the other was used for DNA extraction (D3396, OMEGA, Doraville, GA, USA). The cDNA was synthesized to estimate the effects of overexpression and RNAi BmUGTs through RT-qPCR (reverse transcription-qPCR.). The DNA was used to estimate the relative copy levels of $N$. bombycis infection through qPCR.

\subsection{Statistical Analysis}

One representative data set of three experiments was used to generate figures with GraphPad Prism 8. All statistical analyses were conducted using IBM SPSS v. 22. All results are shown as means $\pm \mathrm{SD}$ of triplicate samples. All data presented are representative of a minimum of three independent experiments.

\section{Results}

\subsection{Identification of the N. bombycis-Inducible BmUGT Genes}

\subsubsection{Transcription of BmUGT Genes in B. mori}

In our previous transcriptome data induced by N. bombycis (unpublished data), the transcription of BmUGT10295 was activated in infected silkworms. In previous research, 42 UGT genes were identified in B. mori, which is much more than that known in other insects, and they belonged to five groups that were identified by phylogenetic analysis. The BmUGT10295 gene belongs to the Group I cluster, which is silkworm-specific [36]. To investigate whether the other B. mori UGT genes were induced by N. bombycis in silkworms, all genes of the Group I cluster were analyzed in infected and uninfected silkworms. The results showed that only the BmUGT10295 and BmUGT8453 genes activated transcription in infected silkworms (Figure 1A).

The transcriptional activation of BmUGT10295 and BmUGT8453 genes in different silkworm tissues showed that there was no transcription in any tissues of uninfected silkworm (Figure 1B). There was also no transcriptional activation in any stages of uninfected silkworms (Figure 1C). These findings indicate that BmUGT10295 and BmUGT8453 genes are the only UGT genes activated by N. bombycis [36].

\subsubsection{Transcription of BmUGT10295 and BmUGT8453 Genes in Different $N$. bombycis-Infected B. mori Tissues}

Although the BmUGT10295 and BmUGT8453 genes were induced in infected silkworms, their transcription in different tissues during infection is unknown. Using N. bombycis-infected silkworms, the transcriptional levels of B. mori BmUGT10295 and BmUGT8453 genes were analyzed by RT-qPCR in different tissues at $48 \mathrm{~h}$ post-infection. The transcription of the BmUGT10295 and BmUGT8453 genes was detected, and mainly in the midgut and Malpighian tubule (Figure 2). According to the MIQE guidelines [47], the primer amplification efficiencies were also carried out. The results showed amplification efficiencies of BmUGT10295 and BmUGT8453 were 105\% and 103\% respectively, which is generally considered acceptable (Figure S1). 
A
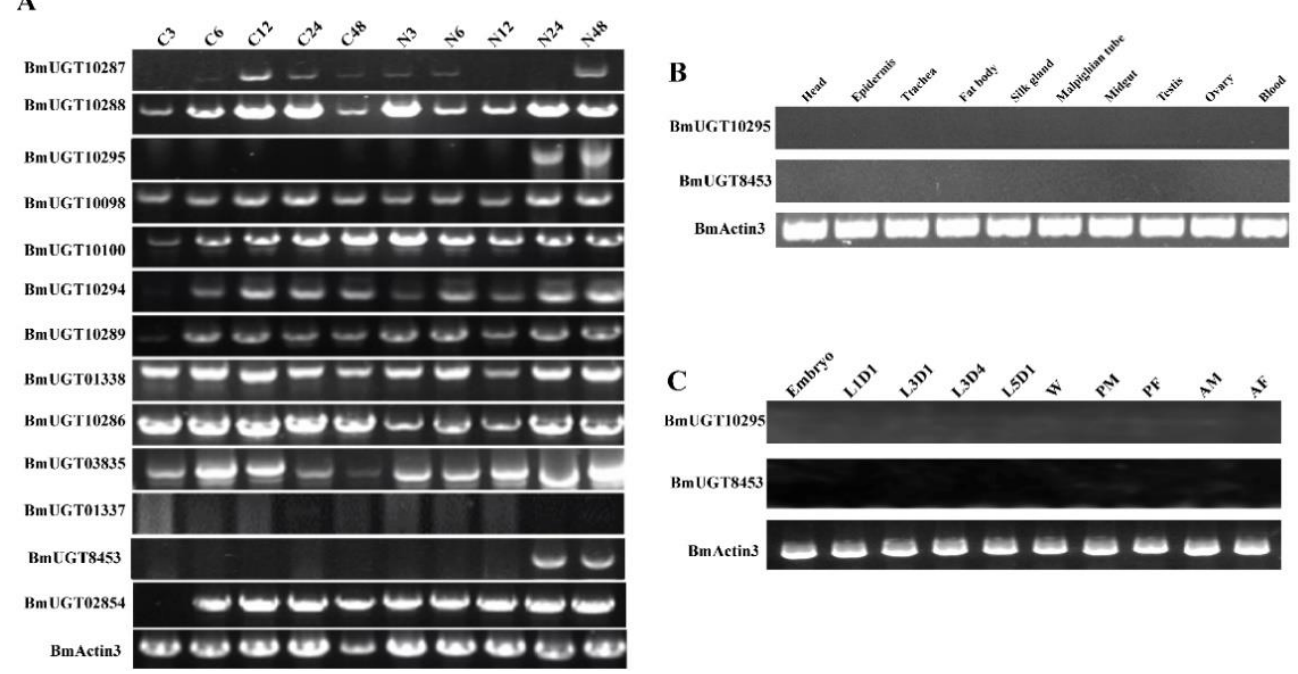

Figure 1. Transcription of Nosema bombycis-inducible BmUGT genes. (A): Different expression patterns of BmUGTs in response to N. bombycis. N3-N48 represents the silkworm samples at 3 48-h postinfection, whereas C3-C48 represent the control group, which was given water. (B): BmUGT10295 and BmUGT8453 transcription in the different tissues of uninfected silkworms on day 3 fifth instar larvae using actin3 (BmActin3) as internal reference. (C): BmUGT10295 and BmUGT8453 transcription in different larval growth stages. RT-PCR was used to analyze the expression characteristics of BmUGT10295 and BmUGT8453 in embryos, 1-day-old first instar larvae (L1D1) to 1-day-old fifth instar larvae (L5D1), wandering larvae (W), male and female pupae (PM/PF), and male and female adults (AM/AF) of uninfected B. mori.
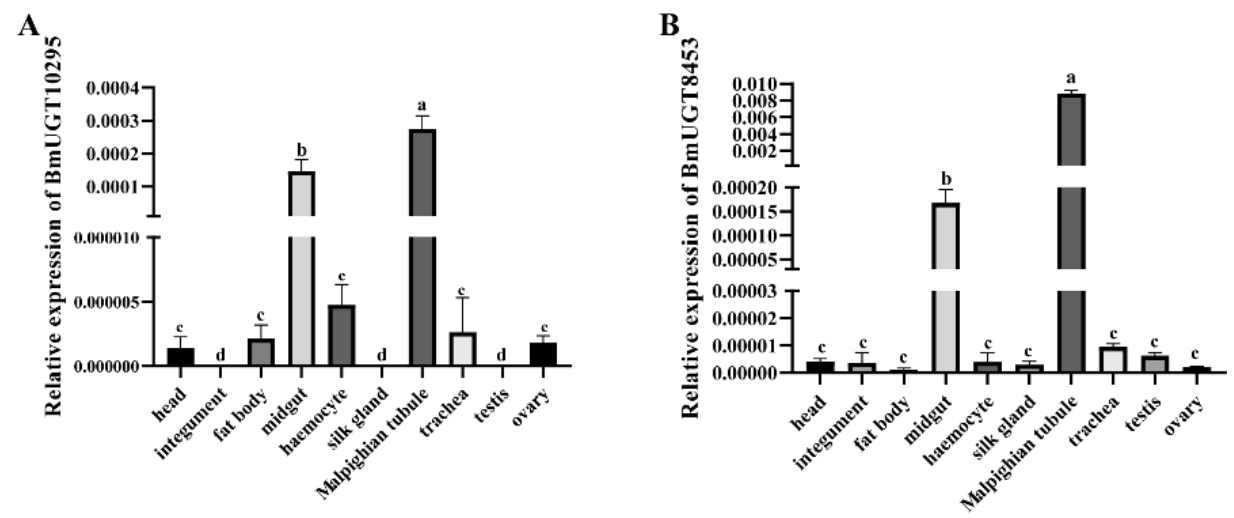

Figure 2. Spatial expression patterns of infected B. mori. Day 1 fifth instar Bombyx mori that were infected with Nosema bombycis. The relative expressions of BmUGT10295 (A) and BmUGT8453 (B) were assessed in different tissues of silkworms 3 days after infection. The relative gene expression levels were estimated according to the $2^{-\Delta C t}$ method. sw22934 was used as an internal reference. Bars represent the mean of three individual measurements \pm SD. Statistical analysis was conducted by one-way ANOVA using a Tukey's multiple comparison test. Identical letters indicate no significant difference $(p>0.01)$, whereas different letters indicate a significant difference $(p<0.01)$.

\subsection{Full ORF Clone of the BmUGT Genes}

Although the BmUGT10295 gene was identified, the sequence information it contains remains controversial [36,37]. The predicted DNA sequence of BmUGT10295 was $780 \mathrm{bp}$ and composed of four exons that encode 260 amino acids, as inferred in the InsectBase database (http: / / www.insect-genome.com/ (accessed on 20 March 2017)) (Figure 3Aa). According to this sequence, the $3^{\prime}$ end of BmUGT10295 was cloned by $3^{\prime}$ RACE (Figure 3B) and the $3^{\prime}$ end sequence was acquired by sequencing. Unfortunately, $5^{\prime}$ RACE failed. Then, the transcription start site (TSS) of BmUGT10295 was predicted (Figure 3Aa). Based on these 
predicted TSS sites, the primers were designed and PCR was performed. The PCR result showed that there were significant amplification bands in TSS-3, TSS-4 and ATG group (BmA3 was used as a control) (Figure 3C). Then, the PCR products were purified, cloned into a PESI vector, and sequenced. The clone and sequence results of TSS-3 were consistent with the genome sequence (Figure 3D). So, the transcriptional initiation of BmUGT10295 was TSS-3. Therefore, the full cDNA of the BmUGT10295 gene was obtained, which was $1550 \mathrm{bp}$ and composed of five exons that encode 271 amino acids (Figure 3Ab,D). Moreover, multiple sequence alignment showed that BmUGT10295, BmUGT8453, and BmUGT1 were highly conserved (Figure S2). Because the sequence of BmUGT8453 gene in KAIKObase was full-length cDNA, the BmUGT8453 gene was also cloned, which the sequencing result was coincident with KAIKObase data.

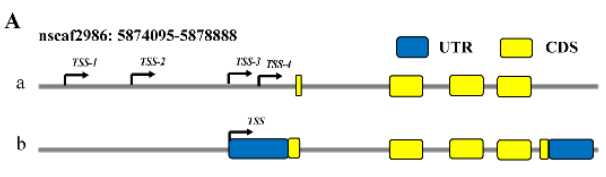

B

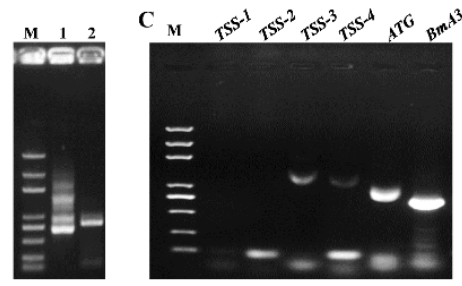

D

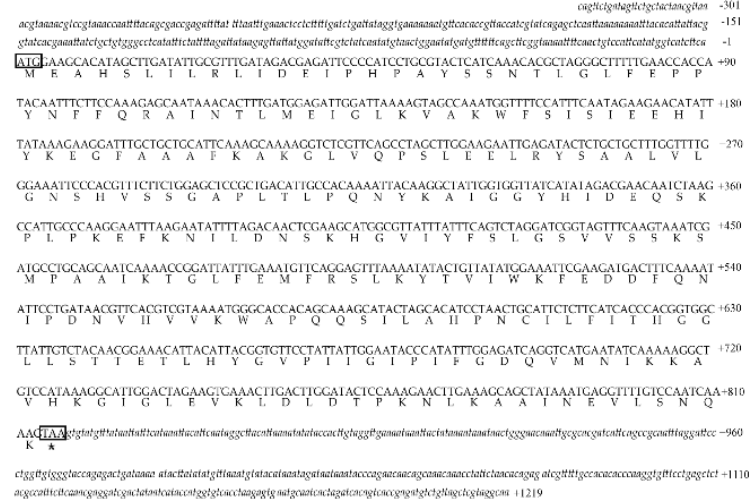

Figure 3. Obtaining the BmUGT10295 full-length cDNA. (A): The schematic phase included structural information of BmUGT10295 in the genome. (a), Annotation information of BmUGT10295 in genome; TSS-1 TSS-4 represented the predicted transcription initiation site; (b), Schematic diagram of BmUGT10295 gene structural information corrected according to experimental results. The yellow squares represent coding DNA sequences (CDS), whereas the blue squares represent untranslated regions (UTR). (B): $3^{\prime}$ RACE of BmUGT10295; 1 is from the first round of PCR and 2 is from the nested PCR. (C): Determination of transcription start sites (TSSs). (D): The full-length cDNA sequence of BmUGT10295.

\subsection{Recombinant BmUGT Purification and Immunoblot Analysis}

The above results showed that BmUGT10295 and BmUGT8453 transcription was detected, but their expression was unknown. Because the DNA sequences of BmUGT10295 and BmUGT8453 are very similar (Figure S2), the differential parts of BmUGT10295 and $B m U G T 8453$ genes were successfully integrated into the pET-32a vector for protein expression in E. coli. SDS-PAGE analysis showed that recombinant BmUGTs (rBmUGTs) were expressed at a molecular mass of $\sim 40 \mathrm{kDa}$, which was consistent with the predicted size (Figure S3A,B). The purified target proteins were cut from the gel and used to prepare the antibody (Figure S3C). Western blot indicated that the rBmUGT antisera specifically recognized an approximately 40-kDa protein (Figure S3D). Then, the antisera of BmUGTs were used to detect the BmUGT10295 and BmUGT8453 expression. The results showed that there were only blot signals in infected silkworms. Expressed BmUGT8453 had a molecular mass of $\sim 60 \mathrm{kDa}$, which was consistent with the predicted size. Additionally, expressed BmUGT10295 had a molecular mass of $\sim 30 \mathrm{kDa}$, which was also consistent with its predicted size and indicated that the ORF was full-length (Figure 4). 


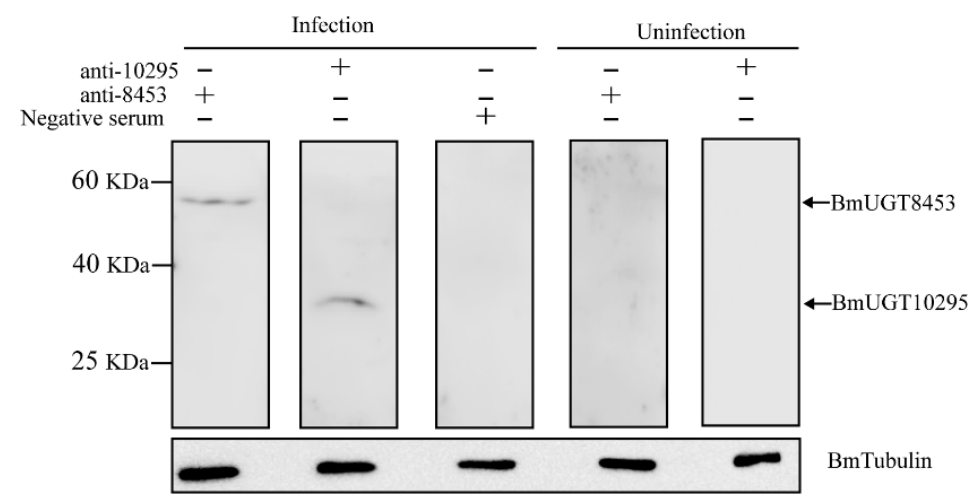

Figure 4. Western blotting analysis of BmUGT expression in infected silkworms. The polyclonal antibodies of BmUGT10295 (anti-10295) and BmUGT8453 (anti-8453) recognized the corresponding signal in infected silkworms, but not in uninfected silkworms. BmTubulin was used as an internal reference.

\subsection{Nosema Bombycis Inhibited by BmUGT10295 and BmUGT8453}

Because BmUGT10295 and BmUGT8453 are induced by N. bombycis, more research was needed to elucidate the role of BmUGT10295 and BmUGT8453 in response to infection. Therefore, a cell-induced expression model was established in the BmN-SWU1 cell line. The results showed that BmUGT10295 and BmUGT8453 were only activated in infected cells (Figure 5).

A

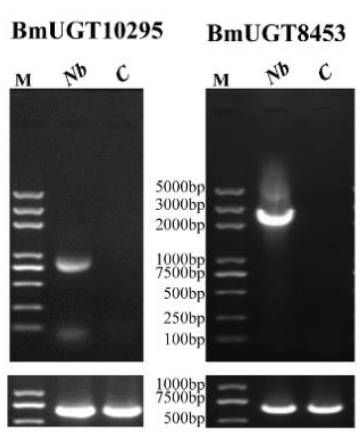

$\mathbf{B}$

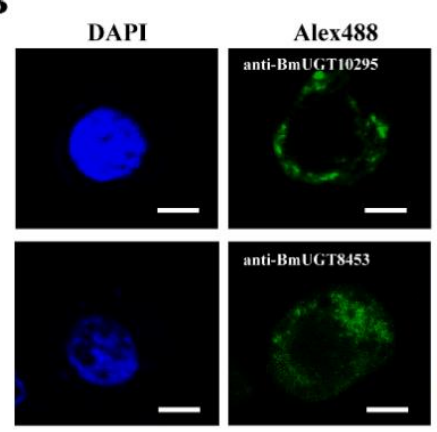

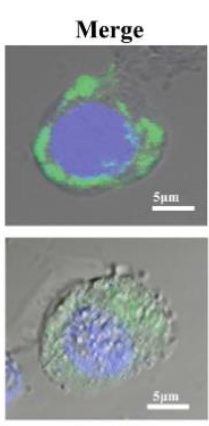

Figure 5. BmUGTs induced by Nosema bombycis in the infected BmN-SWU1 cell line. (A): RT-PCR showed that the BmUGT expression was activated by $N$. bombycis $(\mathrm{Nb})$ in the infected cell line but not in control. Bombyx mori actin3 (BmA3) was the internal reference. (B): Localization of BmUGTs in the infected BmN-SWU1 cell line. Green fluorescence was observed in the samples incubated with the polyclonal antibodies of BmUGTs. Blue fluorescence represents nuclei labeled with DAPI (Sigma, Saint Louis, MO, USA).

Furthermore, the amount of $N$. bombycis proliferation was evaluated in over-expressing BmUGTs cells. The results showed that the proliferation amount of N. bombycis was significantly lower in over-expressing BmUGT10295 and BmUGT8453 cells than in the control group (over-expressing DsRed cells) (Figure 6A,B). Additionally, the proliferation amount of $N$. bombycis in RNAi BmUGTs in BmN-SWU1 cells was much higher than in the control group (Figure 6C,D). The effects of over-expression and RNAi are shown in Figure S4. 

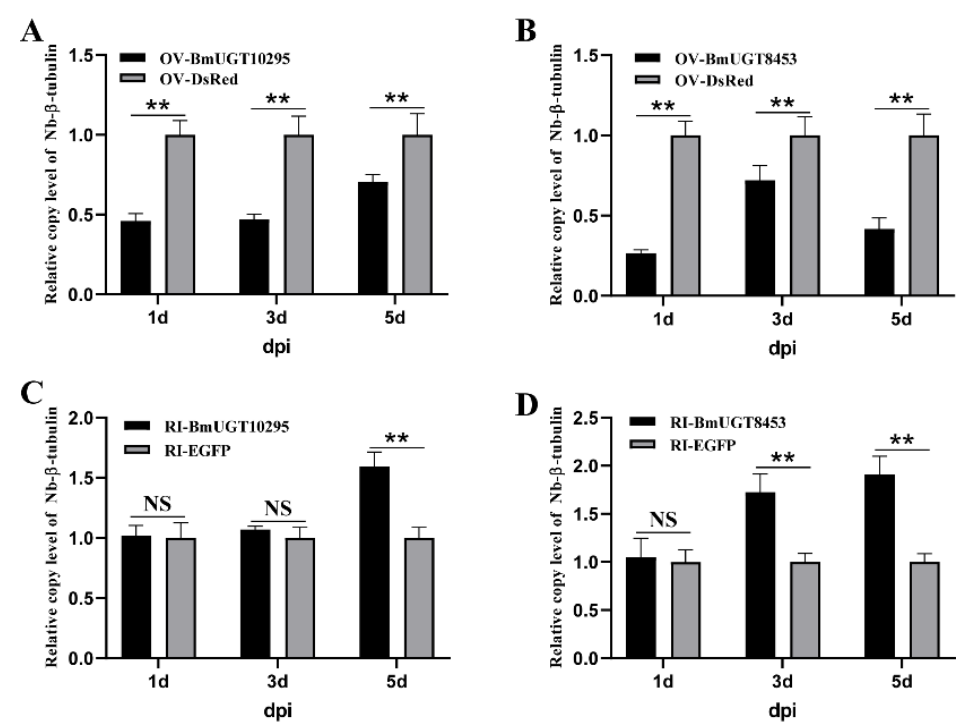

Figure 6. BmUGT10295 and BmUGT8453 inhibited Nosema bombycis infection. The N. bombycis $\beta$-tubulin relative copy levels in over-expressed BmUGT10295 (A) and BmUGT8453 (B) cells were lower than that in over-expressed DsRed cells. The N. bombycis $\beta$-tubulin relative copy levels in RNAi BmUGT10295 (C) and BmUGT8453 (D) cells were higher than that in RNAi EGFP cells. The relative copy levels were estimated according to the $2^{-\Delta \Delta C t}$ method. sw22934 was used as an internal reference. Over-expressed DsRed and RNAi EGFP were used for calibration (value 1). Bars represent the mean of three individual measurements \pm SD. Statistical significance was determined by an unpaired t-test, and statistically significant differences are represented with asterisks $\left({ }^{* *} p<0.01\right)$.

\section{Discussion}

Silkworm, as a model of Lepidoptera, has significant economic and scientific values. Nosema bombycis, a pathogen that causes pebrine disease, can cause severe damage to the sericulture industry. However, few studies have examined the mechanism of host response to microsporidia infection. In previous studies, many genes were found to be induced by N. bombycis that were involved in many signal transduction pathways and are therefore key components of many cellular processes [19,41-44]. Although many genes were found to be activated by N. bombycis through a genome-wide survey in our previous study [41], these genes were determined to have different levels of background expression in subsequent work [48]. In addition, the Hsp70 promoter was reported to have relatively high microsporidia-inducible activity [49]. In this study, BmUGT10295 and BmUGT8453 were screened because they were activated by silkworm post-infection with $N$. bombycis but not in uninfected silkworms (Figure 1).

The sterile insect technique is a highly effective area-wide pest control tool with a low environmental impact, and is primarily used to control mosquitos [50-52] and flies [53]. There have been many sterile insect technique strategies, and one strategy is to use a sex-specific promoter or enhancer to drive the expression of a toxic gene for sexspecific death [54,55]. Microsporidia, an obligate intracellular parasite, must rely on cells to survive. Based on the SIT strategy, a toxic gene, driven by a N. bombyx-induced promoter, expressed explicitly in infected cells, could finally lead to cell death and microsporidia lost its living host. In this way, the silkworm was provided engineer resistance to $N$. bombycis. A previous study showed that an Hsp70 promoter-inducible genome editing system induced resistance to N. bombycis in transgenic silkworms [49], which picked up similar threads. Numerous genes can be up-regulated after microsporidia infection $[41,42,56]$, but few specific microsporidia-inducible genes or promoters were reported. Though most UGTs are constitutively expressed in organisms, in this study, BmUGT10295 and BmUGT8453 were induced after microsporidia infection but had hardly any transcriptional activity in uninfected silkworms (Figure 1). Therefore, the research in this study provides a novel and effective target for promoting silkworm resistance to N. bombycis. 
Members of the UGT superfamily typically catalyze the reaction of the covalent addition of sugar from UDP-sugar cofactors to a lipophilic acceptor, which is a secondorder nucleophilic substitution reaction. UGTs can be divided into two major functional domains [57]. The N-terminal domain, which is variable in sequence between different isoforms, is responsible for binding the aglycone. Alternatively, the C-terminal domain, which is more conserved in sequence, is believed to contain a binding site for the UDP-sugar. For insects, UGTs prominently detoxify xenobiotic compounds from the plant on which they feed. For example, Spodoptera frugiperda uses SfUGT33F28 to inactivate maize defensive benzoxazinoids [58]. Moreover, nicotine, one of the most abundant secondary plant metabolites in tobacco, is highly toxic to herbivorous insects. In Myzus persicae nicotianae, UGTs could be required to detoxify nicotine [22]. In addition to plant xenobiotic tolerance, insect UGTs are also involved in insecticide detoxification. For example, the UGTs of Aphis gossypii are involved in sulfoxaflor [59,60], spirotetramat [24], imidacloprid [61], and other insecticides [62] resistance. four Spodoptera UGT genes are significantly co-upregulated by the lambda-cyhalothrin, chlorantraniliprole, metaflumizone, and indoxacarb insecticides [27]. UGT201D3 is highly expressed and more inducible with abamectin exposure in the abamectin-resistant Tetranychus cinnabarinus strain [63]. Besides response to insecticides, UGT genes are also a response to pathogens. Caenorhabditis elegans UGT29 was robustly induced by Burkholderia pseudomallei [64]. Unlike Caenorhabditis elegans, there are no reports about the involvement of UGT in microbial stress response in insects. In this study, over-expressed or RNAi BmUGTs could affect the number of N. bombycis (Figure 6). Our study is the first report to find that UGT facilitates resistance to pathogens in insects and further analysis is required to reveal this mechanism.

\section{Conclusions}

BmUGT10295 and BmUGT8453 were activated by N. bombycis in infected silkworms. Moreover, these two BmUGTs provided resistance to microsporidia in the BmN-SWU1 cell line. The obtained results contribute to our understanding of host stress reaction to pathogens and provide a novel and effective target for promoting pathogen resistance.

Supplementary Materials: The following are available online at https: / www.mdpi.com/article / 10.3390/insects12090799/s1, Figure S1: The amplification efficiency of primers in BmUGT10295 and BmUGT8453 gene; Figure S2: Multiple alignment of 3 BmUGTs; Figure S3: Purification of rBmUGTs and identification of BmUGTs polyclonal antibodies; Figure S4: The effect evaluations of over-expression and RNAi BmUGTs; Table S1: List of the detected BmUGT genes in B. mori; Table S2: Oligonucleotide primers.

Author Contributions: Conceptualization: Z.Z., C.L., J.W. and G.P.; methodology, validation, formal analysis and investigation: B.Y. and Q.Y.; draft and revision of the manuscript: B.Y. and C.L. All authors have read and agreed to the published version of the manuscript.

Funding: This research was funded by the National Natural Science Foundation of China (Grant number: 32072793) and the Fundamental Research Funds for the Central Universities to Southwest University (XDJK2018AA001).

Institutional Review Board Statement: All animal experiments were conducted in accordance with Laboratory Animals Ethics Review Committee of Southwest University guidelines (Chongqing, China), and the committee approved this study (Permit Number: AERCSWU2017-7).

Informed Consent Statement: Not applicable.

Data Availability Statement: All data are available in the article.

Acknowledgments: We thank Tongbao Liu (State Key Laboratory of Silkworm Genome Biology, Southwest University) for valuable suggestions and editing the draft of this manuscript.

Conflicts of Interest: The authors declare no conflict of interest. 


\section{References}

1. Cavalier-Smith, T. A revised six-kingdom system of life. Biol. Rev. Camb. Philos. Soc. 1998, 73, 203-266. [CrossRef]

2. Pan, G.; Bao, J.; Ma, Z.; Song, Y.; Han, B.; Ran, M.; Li, C.; Zhou, Z. Invertebrate host responses to microsporidia infections. Dev. Comp. Immunol. 2018, 83, 104-113. [CrossRef] [PubMed]

3. Fries, I.; Feng, F.; da Silva, A.; Slemenda, S.B.; Pieniazek, N.J. Nosema ceranae n. sp.(Microspora, Nosematidae), morphological and molecular characterization of a microsporidian parasite of the Asian honey bee Apis cerana (Hymenoptera, Apidae). Eur. J. Protistol. 1996, 32, 356-365. [CrossRef]

4. Zander, E. Tierische parasiten als krankenheitserreger bei der biene. Münchener Bienenztg. 1909, 31, $196-204$.

5. Aranguren Caro, L.F.; Mai, H.N.; Pichardo, O.; Cruz-Flores, R.; Hanggono, B.; Dhar, A.K. Evidences supporting Enterocytozoon hepatopenaei association with white feces syndrome in farmed Penaeus vannamei in Venezuela and Indonesia. Dis. Aquat. Org. 2020, 141, 71-78. [CrossRef]

6. Karthikeyan, K.; Sudhakaran, R. Experimental horizontal transmission of Enterocytozoon hepatopenaei in post-larvae of whiteleg shrimp, Litopenaeus vannamei. J. Fish Dis. 2019, 42, 397-404. [CrossRef]

7. Elkarim Laatamna, A.; Holubova, N.; Sak, B.; Kvac, M. Cryptosporidium meleagridis and C. baileyi (Apicomplexa) in domestic and wild birds in Algeria. Folia Parasitol. 2017, 64. [CrossRef]

8. Shahbazi, P.; Aligolzadeh, A.; Khordadmehr, M.; Hashemzadeh Farhang, H.; Katiraee, F. Molecular study and genotyping of Cryptosporidium baileyi and Cryptosporidium parvum from free-range and commercial broiler chickens in Guilan province, Iran. Comp. Immunol. Microbiol. Infect. Dis. 2020, 69, 101411. [CrossRef]

9. Current, W.L.; Garcia, L.S. Cryptosporidiosis. Clin. Microbiol. Rev. 1991, 4, 325-358. [CrossRef]

10. Didier, E.S.; Weiss, L.M. Microsporidiosis: Not just in AIDS patients. Curr. Opin. Infect. Dis. 2011, 24, 490-495. [CrossRef]

11. Muadica, A.S.; Messa, A.E., Jr.; Dashti, A.; Balasegaram, S.; Santin, M.; Manjate, F.; Chirinda, P.; Garrine, M.; Vubil, D.; Acácio, S.; et al. First identification of genotypes of Enterocytozoon bieneusi (Microsporidia) among symptomatic and asymptomatic children in Mozambique. PLoS Negl. Trop. Dis. 2020, 14, e0008419. [CrossRef]

12. Ghosh, K.; Weiss, L.M. T cell response and persistence of the microsporidia. FEMS Microbiol. Rev. 2012, 36, 748-760. [CrossRef] [PubMed]

13. Moretto, M.M.; Harrow, D.I.; Khan, I.A. Effector CD8 T cell immunity in microsporidial infection: A lone defense mechanism. Semin. Immunopathol. 2015, 37, 281-287. [CrossRef]

14. Szumowski, S.C.; Troemel, E.R. Microsporidia-host interactions. Curr. Opin. Microbiol. 2015, 26, 10-16. [CrossRef]

15. Bhat, S.A.; Bashir, I.; Kamili, A.S. Microsporidiosis of silkworm, Bombyx mori L. (Lepidoptera-Bombycidae): A review. Afr. J. Agric. Res. 2009, 4, 1519-1523.

16. Ni, W.; Bao, J.; Mo, B.; Liu, L.; Li, T.; Pan, G.; Chen, J.; Zhou, Z. Hemocytin facilitates host immune responses against Nosema bombycis. Dev. Comp. Immunol. 2020, 103, 103495. [CrossRef] [PubMed]

17. Zhu, F.; Tang, X.; Xiao, S.; Wang, H.; Zhang, Y.; Shao, Y.; Tang, F.; Chen, S.; Bai, X. The role of Bombyx mori Bmtutl-519 protein in the infection of BmN cells by Nosema bombycis. Dev. Comp. Immunol. 2019, 92, 283-290. [CrossRef] [PubMed]

18. Hua, X.; Xu, W.; Ma, S.; Xia, Q. STING-dependent autophagy suppresses Nosema bombycis infection in silkworms, Bombyx mori. Dev. Comp. Immunol. 2021, 115, 103862. [CrossRef] [PubMed]

19. Tang, X.; Zhang, Y.; Zhou, Y.; Liu, R.; Shen, Z. Quantitative proteomic analysis of ovaries from Nosema bombycis-infected silkworm (Bombyx mori). J. Invertebr. Pathol. 2020, 172, 107355. [CrossRef] [PubMed]

20. He, X.; Fu, Z.; Li, M.; Liu, H.; Cai, S.; Man, N.; Lu, X. Nosema bombycis (Microsporidia) suppresses apoptosis in BmN cells (Bombyx mori). Acta Biochim. Biophys. Sin. 2015, 47, 696-702. [CrossRef]

21. Bock, K.W. Vertebrate UDP-glucuronosyltransferases: Functional and evolutionary aspects. Biochem. Pharmacol. 2003, 66, 691-696. [CrossRef]

22. Pan, Y.; Xu, P.; Zeng, X.; Liu, X.; Shang, Q. Characterization of UDP-Glucuronosyltransferases and the Potential Contribution to Nicotine Tolerance in Myzus persicae. Int. J. Mol. Sci. 2019, 20, 3637. [CrossRef]

23. Krempl, C.; Sporer, T.; Reichelt, M.; Ahn, S.J.; Heidel-Fischer, H.; Vogel, H.; Heckel, D.G.; Joußen, N. Potential detoxification of gossypol by UDP-glycosyltransferases in the two Heliothine moth species Helicoverpa armigera and Heliothis virescens. Insect Biochem. Mol. Biol. 2016, 71, 49-57. [CrossRef] [PubMed]

24. Pan, Y.; Wen, S.; Chen, X.; Gao, X.; Zeng, X.; Liu, X.; Tian, F.; Shang, Q. UDP-glycosyltransferases contribute to spirotetramat resistance in Aphis gossypii Glover. Pestic. Biochem. Physiol. 2020, 166, 104565. [CrossRef]

25. Li, X.; Zhu, B.; Gao, X.; Liang, P. Over-expression of UDP-glycosyltransferase gene UGT2B17 is involved in chlorantraniliprole resistance in Plutella xylostella (L.). Pest Manag. Sci. 2017, 73, 1402-1409. [CrossRef] [PubMed]

26. Cui, X.; Wang, C.; Wang, X.; Li, G.; Liu, Z.; Wang, H.; Guo, X.; Xu, B. Molecular Mechanism of the UDP-Glucuronosyltransferase 2B20-like Gene (AccUGT2B20-like) in Pesticide Resistance of Apis cerana cerana. Front. Genet. 2020, 11, 592595. [CrossRef] [PubMed]

27. Hu, B.; Zhang, S.H.; Ren, M.M.; Tian, X.R.; Wei, Q.; Mburu, D.K.; Su, J.Y. The expression of Spodoptera exigua P450 and UGT genes: Tissue specificity and response to insecticides. Insect Sci. 2019, 26, 199-216. [CrossRef] [PubMed]

28. Zhao, J.; Xu, L.; Sun, Y.; Song, P.; Han, Z. UDP-Glycosyltransferase Genes in the Striped Rice Stem Borer, Chilo suppressalis (Walker), and Their Contribution to Chlorantraniliprole Resistance. Int. J. Mol. Sci. 2019, 20, 1064. [CrossRef] 
29. Nagare, M.; Ayachit, M.; Agnihotri, A.; Schwab, W.; Joshi, R. Glycosyltransferases: The multifaceted enzymatic regulator in insects. Insect Mol. Biol. 2021, 30, 123-137. [CrossRef]

30. Rübsam, R.; Hollmann, M.; Simmerl, E.; Lammermann, U.; Schäfer, M.A.; Büning, J.; Schäfer, U. The egghead gene product influences oocyte differentiation by follicle cell-germ cell interactions in Drosophila melanogaster. Mech. Dev. 1998, 72, 131-140. [CrossRef]

31. Johnson, D.M.; Mugelli, A.; Cerbai, E. Editorial: The Role of Calcium Handling in Heart Failure and Heart Failure Associated Arrhythmias. Front. Physiol. 2019, 10, 1. [CrossRef]

32. Walski, T.; De Schutter, K.; Van Damme, E.J.M.; Smagghe, G. Diversity and functions of protein glycosylation in insects. Insect Biochem. Mol. Biol. 2017, 83, 21-34. [CrossRef]

33. Wiesen, B.; Krug, E.; Fiedler, K.; Wray, V.; Proksch, P. Sequestration of host-plant-derived flavonoids by lycaenid butterflyPolyommatus icarus. J. Chem. Ecol. 1994, 20, 2523-2538. [CrossRef] [PubMed]

34. Bock, K.W. The UDP-glycosyltransferase (UGT) superfamily expressed in humans, insects and plants: Animal-plant arms-race and co-evolution. Biochem. Pharmacol. 2016, 99, 11-17. [CrossRef] [PubMed]

35. Wang, S.; Liu, Y.; Zhou, J.J.; Yi, J.K.; Pan, Y.; Wang, J.; Zhang, X.X.; Wang, J.X.; Yang, S.; Xi, J.H. Identification and tissue expression profiling of candidate UDP-glycosyltransferase genes expressed in Holotrichia parallela motschulsky antennae. Bull. Entomol. Res. 2018, 108, 807-816. [CrossRef] [PubMed]

36. Huang, F.-F.; Chai, C.-L.; Zhang, Z.; Liu, Z.-H.; Dai, F.-Y.; Lu, C.; Xiang, Z.-H. The UDP-glucosyltransferase multigene family in Bombyx mori. BMC Genom. 2008, 9, 563. [CrossRef] [PubMed]

37. Ahn, S.J.; Vogel, H.; Heckel, D.G. Comparative analysis of the UDP-glycosyltransferase multigene family in insects. Insect Biochem. Mol. Biol. 2012, 42, 133-147. [CrossRef]

38. Daimon, T.; Hirayama, C.; Kanai, M.; Ruike, Y.; Meng, Y.; Kosegawa, E.; Nakamura, M.; Tsujimoto, G.; Katsuma, S.; Shimada, T. The silkworm Green b locus encodes a quercetin 5-O-glucosyltransferase that produces green cocoons with UV-shielding properties. Proc. Natl. Acad. Sci. USA 2010, 107, 11471-11476. [CrossRef]

39. Xu, X.; Wang, M.; Wang, Y.; Sima, Y.; Zhang, D.; Li, J.; Yin, W.; Xu, S. Green cocoons in silkworm Bombyx mori resulting from the quercetin 5-O-glucosyltransferase of UGT86, is an evolved response to dietary toxins. Mol. Biol. Rep. 2013, 40, 3631-3639. [CrossRef]

40. Luque, T.; Okano, K.; O'Reilly, D.R. Characterization of a novel silkworm (Bombyx mori) phenol UDP-glucosyltransferase. Eur. J. Biochem. 2002, 269, 819-825. [CrossRef]

41. Ma, Z.; Li, C.; Pan, G.; Li, Z.; Han, B.; Xu, J.; Lan, X.; Chen, J.; Yang, D.; Chen, Q.; et al. Genome-wide transcriptional response of silkworm (Bombyx mori) to infection by the microsporidian Nosema bombycis. PLoS ONE 2013, 8, e84137. [CrossRef]

42. Yue, Y.J.; Tang, X.D.; Xu, L.; Yan, W.; Li, Q.L.; Xiao, S.Y.; Fu, X.L.; Wang, W.; Li, N.; Shen, Z.Y. Early responses of silkworm midgut to microsporidium infection-A Digital Gene Expression analysis. J. Invertebr. Pathol. 2015, 124, 6-14. [CrossRef]

43. Li, Z.; Wang, Y.; Wang, L.; Zhou, Z. Molecular and biochemical responses in the midgut of the silkworm, Bombyx mori, infected with Nosema bombycis. Parasites Vectors 2018, 11, 147. [CrossRef]

44. He, X.; He, X.; Liu, H.; Li, M.; Cai, S.; Fu, Z.; Lu, X. Proteomic analysis of BmN cells (Bombyx mori) in response to infection with Nosema bombycis. Acta Biochim. Biophys. Sin. 2014, 46, 982-990. [CrossRef] [PubMed]

45. Pan, M.H.; Cai, X.J.; Liu, M.; Lv, J.; Tang, H.; Tan, J.; Lu, C. Establishment and characterization of an ovarian cell line of the silkworm, Bombyx mori. Tissue Cell 2010, 42, 42-46. [CrossRef]

46. Yu, B.; Sang, Q.; Pan, G.; Li, C.; Zhou, Z. A Toll-Spätzle Pathway in the Immune Response of Bombyx mori. Insects 2020, 11, 586. [CrossRef] [PubMed]

47. Bustin, S.A.; Benes, V.; Garson, J.A.; Hellemans, J.; Huggett, J.; Kubista, M.; Mueller, R.; Nolan, T.; Pfaffl, M.W.; Shipley, G.L.; et al. The MIQE guidelines: Minimum information for publication of quantitative real-time PCR experiments. Clin. Chem. 2009, 55, 611-622. [CrossRef]

48. Ji, X.; Li, C.; Sun, B.; Zheng, R.; Li, Z.; Chen, J.; Long, M.; Li, T.; Pan, G.; Zhou, Z. Cloning and Expression Analysis of C-type Lectin 11 Gene in Bombyx mori. Sci. Seric. 2016, 042, 627-635. [CrossRef]

49. Dong, Z.; Long, J.; Huang, L.; Hu, Z.; Chen, P.; Hu, N.; Zheng, N.; Huang, X.; Lu, C.; Pan, M. Construction and application of an HSP70 promoter-inducible genome editing system in transgenic silkworm to induce resistance to Nosema bombycis. Appl. Microbiol. Biotechnol. 2019, 103, 9583-9592. [CrossRef] [PubMed]

50. Kittayapong, P.; Ninphanomchai, S.; Limohpasmanee, W.; Chansang, C.; Chansang, U.; Mongkalangoon, P. Combined sterile insect technique and incompatible insect technique: The first proof-of-concept to suppress Aedes aegypti vector populations in semi-rural settings in Thailand. PLoS Negl. Trop. Dis. 2019, 13, e0007771. [CrossRef]

51. Bouyer, J.; Yamada, H.; Pereira, R.; Bourtzis, K.; Vreysen, M.J.B. Phased Conditional Approach for Mosquito Management Using Sterile Insect Technique. Trends Parasitol. 2020, 36, 325-336. [CrossRef]

52. World Health Organization; The International Atomic Energy Agency. Guidance Framework for Testing the Sterile Insect Technique as a Vector Control Tool Against Aedes-Borne Diseases; World Health Organization: Geneva, Switzerland, 2020.

53. Kandul, N.P.; Liu, J.; Sanchez, C.H.; Wu, S.L.; Marshall, J.M.; Akbari, O.S. Transforming insect population control with precision guided sterile males with demonstration in flies. Nat. Commun. 2019, 10, 84. [CrossRef]

54. Thomas, D.D.; Donnelly, C.A.; Wood, R.J.; Alphey, L.S. Insect population control using a dominant, repressible, lethal genetic system. Science 2000, 287, 2474-2476. [CrossRef] 
55. Fu, G.; Lees, R.S.; Nimmo, D.; Aw, D.; Jin, L.; Gray, P.; Berendonk, T.U.; White-Cooper, H.; Scaife, S.; Kim Phuc, H.; et al. Female-specific flightless phenotype for mosquito control. Proc. Natl. Acad. Sci. USA 2010, 107, 4550-4554. [CrossRef] [PubMed]

56. Liu, H.; Li, M.; He, X.; Cai, S.; He, X.; Lu, X. Transcriptome sequencing and characterization of ungerminated and germinated spores of Nosema bombycis. Acta Biochim. Biophys. Sin. 2016, 48, 246-256. [CrossRef] [PubMed]

57. Meech, R.; Mackenzie, P.I. Structure and function of uridine diphosphate glucuronosyltransferases. Clin. Exp. Pharmacol. Physiol. 1997, 24, 907-915. [CrossRef] [PubMed]

58. Israni, B.; Wouters, F.C.; Luck, K.; Seibel, E.; Ahn, S.J.; Paetz, C.; Reinert, M.; Vogel, H.; Erb, M.; Heckel, D.G.; et al. The Fall Armyworm Spodoptera frugiperda Utilizes Specific UDP-Glycosyltransferases to Inactivate Maize Defensive Benzoxazinoids. Front. Physiol. 2020, 11, 604754. [CrossRef]

59. Ma, K.; Tang, Q.; Liang, P.; Li, J.; Gao, X. UDP-Glycosyltransferases from the UGT344 Family Are Involved in Sulfoxaflor Resistance in Aphis gossypii Glover. Insects 2021, 12, 356. [CrossRef]

60. Wang, L.; Zhu, J.; Cui, L.; Wang, Q.; Huang, W.; Yang, Q.; Ji, X.; Rui, C. Overexpression of Multiple UDP-Glycosyltransferase Genes Involved in Sulfoxaflor Resistance in Aphis gossypii Glover. J. Agric. Food Chem. 2021, 69, 5198-5205. [CrossRef]

61. Chen, X.; Xia, J.; Shang, Q.; Song, D.; Gao, X. UDP-glucosyltransferases potentially contribute to imidacloprid resistance in Aphis gossypii glover based on transcriptomic and proteomic analyses. Pestic. Biochem. Physiol. 2019, 159, 98-106. [CrossRef]

62. Chen, X.; Tang, C.; Ma, K.; Xia, J.; Song, D.; Gao, X.W. Overexpression of UDP-glycosyltransferase potentially involved in insecticide resistance in Aphis gossypii Glover collected from Bt cotton fields in China. Pest Manag. Sci. 2020, 76, 1371-1377. [CrossRef] [PubMed]

63. Wang, M.Y.; Liu, X.Y.; Shi, L.; Liu, J.L.; Shen, G.M.; Zhang, P.; Lu, W.C.; He, L. Functional analysis of UGT201D3 associated with abamectin resistance in Tetranychus cinnabarinus (Boisduval). Insect Sci. 2020, 27, 276-291. [CrossRef] [PubMed]

64. Wong, R.R.; Kong, C.; Lee, S.H.; Nathan, S. Detection of Burkholderia pseudomallei toxin-mediated inhibition of protein synthesis using a Caenorhabditis elegans ugt-29 biosensor. Sci. Rep. 2016, 6, 27475. [CrossRef] [PubMed] 